\title{
Guest-editorial
}

\section{Special Issue on Advances in Intelligent Systems}

\author{
J.M. Benítez ${ }^{\mathrm{a}, *}$, Vincenzo Loia ${ }^{\mathrm{b}}$ and Francesco Marcelloni ${ }^{\mathrm{c}}$ \\ ${ }^{a}$ Department of Computer Science and Artificial Intelligence, CITIC-UGR, Universidad de Granada, Granada, \\ Spain \\ ${ }^{\mathrm{b}}$ Department of Mathematics and Computer Science, University of Salerno, Salerno, Italy \\ ${ }^{\mathrm{c}}$ Department of Information Engineering, University of Pisa, Pisa, Italy
}

This special issue consists of five papers focused on recent developments in the field of Intelligent Systems. A worldwide recognized event in this field is the "International Conference on Intelligent Systems, Design and Applications" series, whose last year edition was held in Pisa, Italy, November 30 - December 2, 2009. An assorted list of outstanding contributions to that conference was selected. Their authors expanded the contributions, which subsequently underwent a peer-review process.

The papers included in this issue represent the wide spectrum of techniques and problems addressed within the field of intelligent systems. The reader can find developments based on neural or evolutionary computation, fuzzy systems or rough sets. With respect to the investigated problems, one can find general problems like classification or clustering as well as concrete real-world application issues.

The first paper, "Classification by Evolutionary Generalized Radial Basis Functions," is concerned with classification problems, and proposes a solution based on feed-forward artificial neural networks. Its main contribution is based on the nature of the functions used in the hidden layer. An evolutionary algorithm is developed to achieve the complete learning of the neural network including both the structure and parameters.

The second paper, "Measures for Unsupervised Fuzzy-Rough Feature Selection," is focused on a recurrent important problem: feature selection. This is

\footnotetext{
*Corresponding author. E-mail: J.M.Benitez@ decsai.ugr.es.
}

a key step in any preprocessing task. Its complexity grows in unsupervised contexts. The authors contribute several measures based on Rough Sets to guide feature selection algorithms. They show how algorithms based on these measures can achieve significant reduction in dimension while preserving the semantics of data.

In the third paper, "Evolutionary Algorithms for Relational Clustering," the trek for an underlying structure in relational data is addressed. The considered data is expressed in terms of proximity matrices instead of classic distance functions. Two versions of a powerful evolutionary algorithm are derived and their performance studied in depth.

The fourth paper, "GA-based Solutions Comparison for Storage Strategies Optimization for an Automated Warehouse," illustrates a specific real-world application of intelligent systems. The problem is finding allocation and reordering strategies for an automated warehouse in the steelmaking industry. This is actually a multi-objective problem for which three different approaches are applied and their performance compared.

The fifth paper, "Local-Global Neuro-Fuzzy System for Color Change Modelling," is also targeted at a concrete real-world problem: color change modeling in odonthology. A system based on the well known neurofuzzy approach is proposed. The system is modified to perform a simultaneous optimization at global and local scopes.

As Guest Editors of this special issue, we would like to thank all the authors for their contributions, and the referees for their necessary, disinterested, and outstanding cooperation as well as for their constructive feedback. 